were VGI, including a cluster of isolates with a single multilocus sequence type originating in the southeastern United States (10). The isolate in this case was identified as molecular type VGI and by multilocus sequence typing was shown to have the same sequence type as isolates from patients in Florida and Georgia and isolates from the environment in Washington.

Infections attributable to $C$. gattii are not confined to tropical and subtropical regions. The case we describe serves to extend the known range of this organism to include Illinois. Infections might be missed, given that many laboratories do not routinely differentiate $C$. gattii from $C$. neoformans. Mortality rates can range from $13 \%$ to $33 \%$ (4). Thus, clinicians and laboratorians must have increased awareness of this emerging infectious disease.

\section{Acknowledgments}

We thank Shawn Lockhart and Colleen Lysen for help with the genotyping of the isolate.

\section{About the Author}

Dr. Moritz is an infectious disease physician for Franciscan Alliance Physician Network in northwest Indiana and a voluntary faculty member for the Indiana University School of Medicine. Her primary research interests include emerging infectious diseases and infection prevention and control.

\section{References}

1 Chen SC-A, Meyer W, Sorrell TC. Cryptococcus gattii infections. Clin Microbiol Rev. 2014;27:980-1024. http://dx.doi.org/10.1128/ CMR.00126-13

2. Espinel-Ingroff A, Kidd SE. Current trends in the prevalence of Cryptococcus gattii in the United States and Canada. Infect Drug Resist. 2015;8:89-97. http://dx.doi.org/10.2147/IDR.S57686

3. Hoang L, Philips P, Galanis E. Cryptococcus gattii: a review of the epidemiology, clinical presentation, diagnosis, and management of this endemic yeast in the Pacific Northwest. Clin Microbiol Newsl. 2011;33:187-95. http://dx.doi.org/10.1016/ j.clinmicnews.2011.11.003

4. CDC. C. gattii infection statistics [cited 2017 Oct 10]. https://www.cdc.gov/fungal/diseases/cryptococcosis-gattii/ statistics.html

5. Harris JR, Lockhart SR, Sondermeyer G, Vugia DJ, Crist MB, D'Angelo MT, et al. Cryptococcus gattii infections in multiple states outside the US Pacific Northwest. Emerg Infect Dis. 2013;19:1620-6. http://dx.doi.org/10.3201/eid1910.130441

6. Viereck MJ, Chalouhi N, Krieger DI, Judy KD. Cryptococcal ventriculoperitoneal shunt infection. J Clin Neurosci. 2014;21:2020-1. http://dx.doi.org/10.1016/j.jocn.2014.08.001

7. Ingram CW, Haywood HB III, Morris VM, Allen RL, Perfect JR. Cryptococcal ventricular-peritoneal shunt infection: clinical and epidemiological evaluation of two closely associated cases. Infect Control Hosp Epidemiol. 1993;14:719-22. http://dx.doi.org/10.2307/30148351

8. Mangham D, Gerding DN, Peterson LR, Sarosi GA. Fungal meningitis manifesting as hydrocephalus. Arch Intern Med. 1983; 143:728-31. http://dx.doi.org/10.1001/ archinte.1983.00350040118015

9. Lockhart SR, Iqbal N, Harris JR, Grossman NT, DeBess E, Wohrle R, et al. Cryptococcus gattii in the United States: genotypic diversity of human and veterinary isolates. PLoS One. 2013;8:e74737. http://dx.doi.org/10.1371/ journal.pone. 0074737

10. Lockhart SR, Roe CC, Engelthaler DM. Whole-genome analysis of Cryptococcus gattii, southeastern United States. Emerg Infect Dis. 2016;22:1098-101. http://dx.doi.org/10.3201/eid2206.151455

Address for correspondence: Amanda T. Harrington, Loyola University Medical Center, Clinical Microbiology Laboratory, Pathology, 2160 S. First Ave, Maywood, IL 60153, USA; email: amanda.harrington@lumc.edu

\title{
Late or Lack of Vaccination Linked to Importation of Yellow Fever from Angola to China
}

\section{Rui Song, ${ }^{1}$ Shengcan Guan, ${ }^{1}$ Shui Shan Lee, ${ }^{1}$ Zhihai Chen, Chen Chen, Lifen Han, Yanli Xu, Ang Li, Hui Zeng, Hanhui Ye, Fujie Zhang}

Author affiliations: Beijing Ditan Hospital, Capital Medical University, Beijing, China (R. Song, Z. Chen, Y. Xu, F. Zhang); Mengchao Hepatobiliary Hospital of Fujian Medical University, Fuzhou, China (S. Guan, L. Han, H. Ye); The Chinese University of Hong Kong, Shatin, Hong Kong, China (S.S. Lee); Beijing Key Laboratory of Emerging and Reemerging Infectious Diseases, Beijing (C. Chen, A. Li, H. Zeng)

DOI: https://doi.org/10.3201/eid2407.171868

During March and April 2016, 11 yellow fever cases were identified in China. We report epidemic and viral information for 10 of these patients, 6 of whom had been vaccinated before travel. Phylogenetic analyses suggest these viruses nested within the diversity of strains endemic to Angola, where an outbreak began in 2015.

$I^{\prime}$ December 2015, the first case of a major yellow fever outbreak was reported in Angola; the outbreak spread to Democratic Republic of the Congo (DRC) (http:// www.who.int/csr/don/13-april-2016-yellow-fever-angola/ en/). In addition to 965 confirmed cases in DRC during

${ }^{1}$ These authors contributed equally to this article. 
December 2016-February 2017 (https://reliefweb.int/report/democratic-republic-congo/yellow-fever-outbreakangola-and-democratic-republic-congo-ends), the outbreak led to exportation to other countries. In China, 11 imported cases were reported during March and April 2016 (1); 1 case-patient subsequently died (2). We conducted a clinical-epidemiologic study on 10 of the 11 case-patients. The study was approved by the Review Board of Beijing Ditan Hospital (Beijing, China) and the Ethics Committee of State Key Laboratory of Pathogen and Biosecurity. We also obtained informed consent from recruited patients.

We confirmed the yellow fever diagnoses according to criteria established by the China National Health and Family
Planning Commission, in agreement with the recommendations of the World Health Organization (3). All case-patients were citizens of China who had stayed in Angola for a period of 5 months to 7 years before returning to China; 7 (70\%) were male, the median age was 41 (range 17-50) years (online Technical Appendix Table 1, https://wwwnc.cdc.gov/EID/ article/24/7/17-1868-Techapp1.pdf). Six case-patients reported a history of yellow fever vaccination; 4 of those were vaccinated $<14$ days before symptom onset. Case-patient 7 received vaccination in China 5 years before symptoms occurred, and case-patient 10 was vaccinated in Namibia 10 months before onset of illness. None had received fractional doses of yellow fever vaccine as advocated in some studies (4).

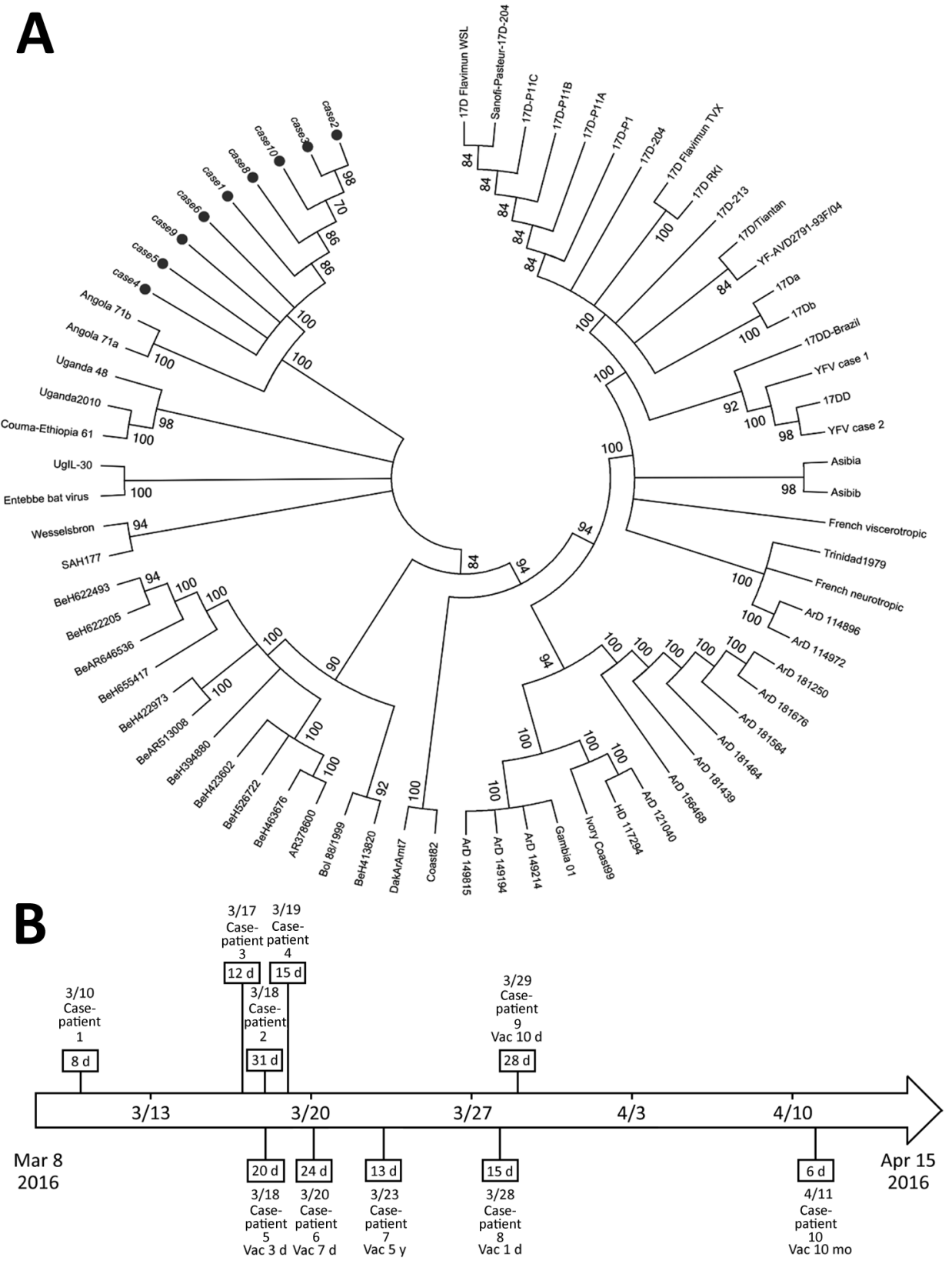

Figure. Phylogenetic analysis of yellow fever viruses and clinical courses for persons with yellow fever cases imported from Angola to China. A) Phylogenetic relationships among the yellow fever viruses from samples obtained from 9 case-patients (black circles). An unrooted dendrogram with maximumlikelihood by genome sequences represents the phylogenetic relationships. Clusters with bootstrap support values $<70$ were integrated; bootstrap values are shown on the branches. B) Dates of hospital admission, detection of virus in urine, and vaccination status for the 10 case-patients investigated. Case-patients 1-4 were not vaccinated. Numbers in boxes indicate longest interval from symptom onset to urine virus sequence detection. Vac, interval from previous vaccination to symptom onset. 
We tested blood samples from all case-patients for yellow fever-specific antibodies by ELISA. We also extracted RNA from blood and urine samples by using the QIAamp MinElute Virus Spin Kit (QIAGEN, Valencia, CA, USA) for reverse transcription PCR virus detection. Phylogenetic analysis (Figure, panel A) of samples from 9 case-patients showed that the sequences were very closely related to each other and resembled the wild-type strain implicated in the Angola outbreak (2). We evaluated virus sequences of 5 of the 6 vaccinated case-patients; all showed the same wild-type strain. For the remaining patient (case-patient 7), RNA extraction was unsuccessful. She had been vaccinated 5 years before her illness, excluding the possibility of a vaccine-related adverse event.

Clinically, all 10 case-patients had acute onset of fever lasting 1-7 days; the highest temperature was $39.5^{\circ} \mathrm{C}$ (online Technical Appendix Table 2). The most common symptoms were fatigue, headache, dizziness, and myalgia. Of 10 case-patients, 8 had been treated for malaria before yellow fever was confirmed. Eight case-patients, including 2 of the 4 unvaccinated case-patients, had relatively mild symptoms. Case-patients 1 and 2, both unvaccinated, had severe disease; signs and symptoms were jaundice, vomiting, hemorrhaging (petechiae, ecchymosis, and gastrointestinal bleeding), and oliguria, as well as high levels of liver enzymes (alanine aminotransferase 11,425 and 3,710 u/L, respectively) and total bilirubin $(>100 \mu \mathrm{mol} / \mathrm{L})$. We also noted bleeding tendency, reflected by a high international normalized ratio and thrombocytopenia. Case-patient 1 deteriorated rapidly as a result of severe kidney and liver damage; biopsy showed evidence of panlobular and confluent hepatocyte necrosis (2). His CD4 T cell count was low at 155 cells/ $\mu \mathrm{L}$, but we excluded HIV co-infection. Despite continuous hemofiltration and hemodialysis and mechanical ventilation support, he died 9 days after symptom onset. The remaining 9 patients recovered after hospitalization, which lasted for a median of 16 (range 11-52) days.

Case-patients 1 and 2 also showed strong inflammatory responses as reflected by high plasma level of interleukin 6. Yellow fever virus nucleic acid continued to be detectable in urine during week 1 after symptom onset; case-patient 2 had the longest interval of detection at 31 days (Figure, panel B).

Overall, the clinical courses of these imported yellow fever cases in China were similar to others reported in the published literature: mild diseases in most case-patients, but high fatality rates among severe cases (5). China is not a yellow fever-endemic area, but importation of the virus was not surprising, considering the high number of travelers between Angola and China (6). Unfortunately, vaccination coverage was not high for Chinese travelers bound for Angola; among this cohort, only 2 had been vaccinated before travel to a yellow fever-endemic area. Travelers may be worried about vaccine-associated viscerotropic disease (7), but this condition did not occur among the vaccinated case-patients in our study, who all had a moderate disease, requiring hospitalization of 11-29 days. The World Health Organization had recommended vaccination at least 10 days before entering a yellow fever-endemic area, but late vaccination in travelers is common (8).

Our observations highlight the importance of timely immunization to achieve protection during an outbreak within a yellow fever-endemic area. Vaccination efficacy and long-term protection are other concerns highlighted in this study: 2 patients were infected despite vaccination received 1-5 years previously. Although most international guidelines did not recommend booster administration of vaccine, its possible role in outbreak settings demands further research $(9,10)$.

\section{Acknowledgments}

The authors acknowledge the technical support of Li Ka Shing Institute of Health Sciences.

This study was supported by Beijing Municipal Science \& Technology Commission (grant no.: Z161100000116049).

\section{About the Author}

Dr. Rui is an infectious disease physician of Medicine Clinical and Research Center of Infectious Diseases at Beijing Ditan Hospital, Capital Medical University in Beijing, China. Her research interests include emerging and reemerging infectious disease.

\section{References}

1. Wang L, Zhou P, Fu X, Zheng Y, Huang S, Fang B, et al. Yellow fever virus: increasing imported cases in China. J Infect. 2016;73:377-80. 10.1016/j.jinf.2016.07.003 http://dx.doi.org/ 10.1016/j.jinf.2016.07.003

2. Chen Z, Liu L, Lv Y, Zhang W, Li J, Zhang Y, et al. A fatal yellow fever virus infection in China: description and lessons. Emerg Microbes Infect. 2016;5:e69. 10.1038/emi.2016.89 http://dx.doi.org/10.1038/emi.2016.89

3. World Health Organization. Dept. of Vaccines and Biologicals. (2003). WHO-recommended standards for surveillance of selected vaccine-preventable diseases. Geneva: WHO, 2003 (revised 2008). http://apps.who.int/iris/bitstream/handle/10665/ 68334/WHO_V-B_03.01_eng.pdf? sequence $=1$ \&isAllowed $=y$

4. World Health Organization. WHO position on the use of fractional doses - June 2017, addendum to vaccines and vaccination against yellow fever WHO: position paper - June 2013. Vaccine October 2017; [Epub ahead of print: Jul 6 2017] http://dx.doi.org/10.1016/ j.vaccine.2017.06.087

5. Monath TP. Yellow fever: an update. Lancet Infect Dis. 2001;1:1120. http://dx.doi.org/10.1016/S1473-3099(01)00016-0

6. Wilder-Smith A, Leong WY. Importation of yellow fever into China: assessing travel patterns. J Travel Med. 2017;24.10.1093/ $\mathrm{jtm} / \operatorname{tax} 008 \mathrm{http}: / /$ dx.doi.org/10.1093/jtm/tax008

7. Beck AS, Barrett AD. Current status and future prospects of yellow fever vaccines. Expert Rev Vaccines. 2015;14:1479-92. 10.1586/14760584.2015 http://dx.doi.org/10.1586/ 14760584.2015 .1083430 
8. Krief I, Goldblatt JG, Paz A, Potasman I. Late vaccination against yellow fever of travelers visiting endemic countries. Travel Med Infect Dis. 2006;4:94-8. http://dx.doi.org/10.1016/ j.tmaid.2005.02.001

9. Gotuzzo E, Yactayo S, Córdova E. Efficacy and duration of immunity after yellow fever vaccination: systematic review on the need for a booster every 10 years. Am J Trop Med Hyg. 2013;89:434-44. 10.4269/ajtmh.13-0264 http://dx.doi.org/10.4269/ ajtmh.13-0264

10. Collaborative group for studies on yellow fever vaccines. Duration of post-vaccination immunity against yellow fever in adults. Vaccine. 2014;39:4977-84. PubMed https://doi.org/ 10.1016/j.vaccine.2014.07.021

Address for correspondence: Fujie Zhang and Hanhui Ye, Beijing Ditan Hospital, Beijing 100015, China; email: treatment@chinaaids.cn, and yehanhui@163.com

\section{Enterovirus A71 Infection, Thailand, 2017}

\section{Jiratchaya Puenpa, Chompoonut Auphimai, Sumeth Korkong, Sompong Vongpunsawad, Yong Poovorawan}

Author affiliation: Chulalongkorn University, Bangkok, Thailand

DOI: https://doi.org/10.3201/eid2407.171923

An outbreak of hand, foot and mouth disease among children in Thailand peaked in August 2017. Enterovirus A71 subgenogroup B5 caused most $(33.8 \%, 163 / 482)$ cases. Severe disease (myocarditis and encephalitis) was observed in 1 patient. Coxsackievirus $A 6$ was detected in $6.0 \%(29 / 482)$ of patients, and coxsackievirus A16 was detected in $2.7 \%(13 / 482)$ of patients.

$\mathrm{H}$ and, foot and mouth disease (HFMD) and herpangina, caused primarily by enterovirus A, commonly affect children and result in painful blisters in the buccal cavity and on the soles of the hands and feet. In rare situations, enterovirus infection can lead to severe neurologic complications, notably aseptic meningitis, encephalitis, acute flaccid paralysis, and death in young children (1).

A nationwide outbreak of HFMD caused by coxsackievirus A6 (CV-A6) affected many children in Thailand in 2012 (2). Since that time, $\approx 50,000$ cases of HFMD have been reported annually to the Thailand Ministry of Public Health (3); CV-A6 and CV-A16 are the main causative agents. Most reports were based on clinical symptoms; laboratory-based confirmations are rare. Consequently, the incidence increased awareness and the need for diagnostic-based epidemiologic surveillance. As of September 25, 2017, a total of 59,071 cases of HFMD have been reported (4). We determined the prevalence and viral etiology of enterovirus infections among patients with clinical HFMD and herpangina in Thailand during 2017.

The study was approved by the Chulalongkorn University Faculty of Medicine Institutional Review Board (institutional review board no. 002/60). We tested clinical specimens from 482 children requiring hospital care for HFMD $(\mathrm{n}=435)$ and herpangina $(\mathrm{n}=47)$ that were submitted to King Chulalongkorn Memorial Hospital (Bangkok, Thailand) during January 1-October 30, 2017, from 12 provinces with reported HFMD outbreaks (online Technical Appendix Figure 1, https://wwwnc.cdc.gov/EID/article/24/7/17-1923Techapp1.pdf). The study population was 273 boys and 209 girls (sex ratio M:F 1.3:1; age range 4 days to 45 years; mean \pm SD age $2.9 \pm 4.0$ years; median age 2.0 years).

We subjected specimens to 2 real-time reverse transcription PCRs (RT-PCRs). The first RT-PCR identifies enterovirus A71 (EV-A71), CV-A6, and CV-A16 (5). We then subjected virus-positive samples to full-length virus capsid protein 1 (VP1) gene amplification by using a conventional RT-PCR and nucleotide sequencing to identify EV-A71 subgenogroups (6). The second RT-PCR is a panenterovirus real-time RT-PCR, which also detects the glyceraldehyde-3-phosphate dehydrogenase gene (internal control). We subjected samples to a conventional RT-PCR that used CODEHOP degenerate primers to identify enterovirus serotypes other than EV-A71, CV-A6, and CV-A16 (7). We also performed molecular typing by using phylogenetic analysis and nucleotide sequence comparisons of strains. Sequences were deposited in GenBank (accession nos. MG843892-844136).

Enterovirus infections were detected throughout the year; increased frequency was observed in the rainy season (June-September) (online Technical Appendix Figure 1). Enteroviruses were identified in $67.4 \%(325 / 482)$ of samples. EV-A71 was most frequent $(33.8 \%, 163 / 482)$, followed by CV-A6 $(6.0 \%, 29 / 482)$, and CV-A16 $(2.7 \%, 13 / 482)$. Analysis of partial VP1 sequences showed that almost all (99.4\%, 162/163) of EV-A71 was subgenogroup B5: only 2 samples were subgenogroup C4 (Figure 1A). Full-length VP1 analysis also confirmed our EV-A71 subgenogroup assignment (online Technical Appendix Figure 2).

Comparison of full-length VP1 nucleotide sequences of subgenogroup B5 viruses between strains isolated in Thailand during this study and those previously isolated in 